\title{
GPS observations of medium-scale traveling ionospheric disturbances over Europe
}

\author{
Y. Otsuka ${ }^{1}$, K. Suzuki ${ }^{1}$, S. Nakagawa ${ }^{1}$, M. Nishioka ${ }^{1,2}$, K. Shiokawa ${ }^{1}$, and T. Tsugawa ${ }^{2}$ \\ ${ }^{1}$ Solar-Terrestrial Environment Laboratory, Nagoya University, Nagoya 464-8601, Japan \\ ${ }^{2}$ National Institute of Information and Communications Technology, 4-2-1 Nukui-Kitamachi, Koganei, Tokyo 184-8795, \\ Japan
}

Correspondence to: Y. Otsuka (otsuka@stelab.nagoya-u.ac.jp)

Received: 26 July 2012 - Revised: 3 January 2013 - Accepted: 4 January 2013 - Published: 5 February 2013

\begin{abstract}
Two-dimensional structures of medium-scale traveling ionospheric disturbances (MSTIDs) over Europe have been revealed, for the first time, by using maps of the total electron content (TEC) obtained from more than 800 GPS receivers of the European GPS receiver networks. From statistical analysis of the TEC maps obtained 2008, we have found that the observed MSTIDs can be categorized into two groups: daytime MSTID and nighttime MSTID. The daytime MSTID frequently occurs in winter. Its maximum occurrence rate in monthly and hourly bin exceeds $70 \%$ at lower latitudes over Europe, whereas it is approximately $45 \%$ at higher latitudes. Since most of the daytime MSTIDs propagate southward, we speculate that they could be caused by atmospheric gravity waves in the thermosphere. The nighttime MSTIDs also frequently occur in winter but most of them propagate southwestward, in a direction consistent with the theory that polarization electric fields play an important role in generating the nighttime MSTIDs. The nighttime MSTID occurrence rate shows distinct latitudinal difference: The maximum of the occurrence rate in monthly and hourly bin is approximately $50 \%$ at lower latitudes in Europe, whereas the nighttime MSTID was rarely observed at higher latitudes. We have performed model calculations of the plasma density perturbations caused by a gravity wave and an oscillating electric field to reproduce the daytime and nighttime MSTIDs, respectively. We find that TEC perturbations caused by gravity waves do not show dip angle dependencies, while those caused by the oscillating electric field have a larger amplitude at lower latitudes. These dip angle dependencies of the TEC perturbation amplitude could contribute to the latitudinal variation of the MSTID occurrence rate. Comparing with previous studies, we discuss the longi-
\end{abstract}

tudinal difference of the nighttime MSTID occurrence rate, along with the E- and F-region coupling processes. The seasonal variation, of the nighttime MSTID occurrence rate in Europe, is not consistent with the theory that the longitudinal and seasonal variations of the nighttime MSTID occurrence could be attributed to those of the Es layer occurrence.

Keywords. Ionosphere (Ionospheric disturbances)

\section{Introduction}

In the mid-latitude ionosphere, traveling ionospheric disturbances (TIDs), which are wave-like structures of plasma density, have been observed by using various techniques (e.g., Hocke and Schlegel, 1996). Since the work of conducted by Hines (1960), TIDs have been believed to be an ionospheric manifestation of atmospheric gravity waves probably caused by geomagnetic activity at high latitude and/or propagated from the lower atmosphere. TIDs with horizontal wavelengths of $100-250 \mathrm{~km}$ are classified as medium-scale TIDs (MSTIDs) (Hunsucker, 1982).

Saito et al. (1998) were the first to show two-dimensional maps of total electron content (TEC) perturbations caused by MSTIDs over Japan using a dense global positioning system (GPS) network consisting of about 1000 GPS receivers. Kotake et al. (2007) and Otsuka et al. (2011) have applied this method to the TEC data obtained from the GPS receiver networks in Southern California and Japan, respectively, and disclosed statistical characteristics of the MSTIDs. Southwestward propagation of the nighttime MSTIDs observed with the GPS receiver networks is consistent with the MSTIDs observed in 630-nm airglow images (e.g., Garcia 
et al., 2000; Martinis et al., 2010; Shiokawa et al., 2003a). Ogawa et al. (2002) have compared spatial structures of the MSTIDs in the GPS TEC map and the 630-nm airglow image and have shown good coincidence between enhancements in the 630-nm airglow image and the GPS TEC. Shiokawa et al. (2003a) and Martinis et al. (2010) have reported statistical characteristics of nighttime MSTIDs over Arecibo in Puerto Rico and Rikubetsu and Shigaraki in Japan, respectively. They have reported that most nighttime MSTIDs propagate southwestward. Miller et al. (1997) and Kelley and Miller (1997) pointed out that this preferred propagation direction cannot be explained by the classical theory of atmospheric gravity waves alone and suggested that electrodynamical forces could play an important role in generating the nighttime MSTIDs. The Perkins instability is one of the possible mechanisms generating the nighttime MSTIDs (Perkins, 1973) because the preferred alignment (NW-SE and NE-SW in the Northern and Southern Hemispheres, respectively) of the MSTID wavefronts is consistent with the condition that the Perkins instability grows in time. However, the linear growth rate of the Perkins instability is too small to explain the observational results (e.g., Kelley and Makela, 2001). Tsunoda and Cosgrove (2001) and Kelley et al. (2003) have suggested that the electrodynamical coupling between E- and F-regions could cause positive feedback, thereby enhancing the polarization electric fields and displacement of the plasma density distribution in the Eand F-regions. Saito et al. (2007) and Otsuka et al. (2007) have shown that both nighttime MSTIDs and E-region irregularities over Japan have wavy structures with a NW-SE aligned wavefront propagating southwestward, and they suggested that the electrodynamical coupling between the Es layer and F-region could play an important role in generating both nighttime MSTIDs and E-region irregularities. By developing a three-dimensional numerical model, Yokoyama et al. (2009) and Yokoyama and Hysell (2010) have simulated the coupling process between the E- and F-regions and suggested that the polarization electric fields driven by the neutral winds in the E-region are essentially important for the development of the Perkins instability in the F-region.

Using the worldwide GPS receiver network, Kotake et al. (2006) have investigated the MSTID occurrence rate in six regions located at mid-latitudes and have shown that the occurrence rate of nighttime MSTIDs depends on longitude. Park et al. (2010) have analyzed plasma density data obtained by satellites and reported the global distribution of the MSTID occurrence rate. They have pointed out that these longitudinal and seasonal variations of the MSTID occurrence rate could be controlled by those of the Es layer appearance. Therefore, a study of the longitudinal dependence of the nighttime MSTID occurrence may provide important information regarding the E- and F-region electrodynamical coupling processes.

In the present paper, two-dimensional maps of TEC perturbations caused by MSTIDs over Europe are presented for

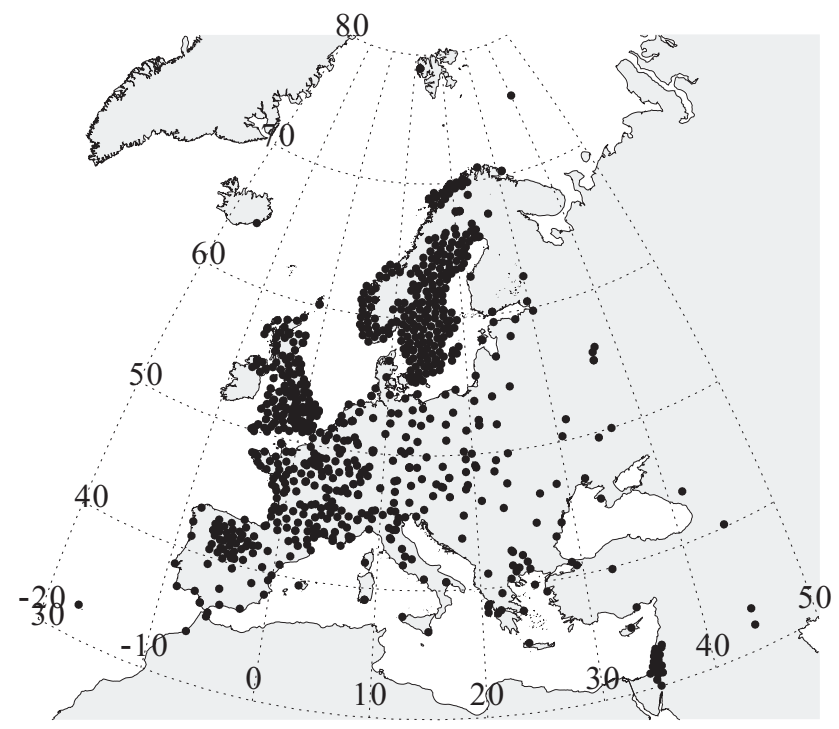

Fig. 1. Location of the GPS receivers in Europe.

the first time. We also report statistical characteristics of the MSTIDs over Europe in 2008, and discuss possible mechanisms causing the MSTIDs.

\section{Observations}

In Europe, more than 800 permanent GPS receivers have been installed as of 2009. The data from these GPS receivers are provided by several different institutes: International GNSS Service (IGS), EUREF Permanent Network (EPN), Federal Agency for Cartography and Geodesy (BKGE) in Germany, Austrian Academy of Sciences, Space Research Institute (OLG) in Austria, National Geographic Institute (IGNE) in France, Delft University of Technology (DUT) in The Netherlands, Agenzia Spatiale Italiana (ASI) in Italy, Agrarian Technological Institute of Castilla and Leon (ITACYL) in Spain, European Sea-Level Service (ESEAS), SWEPOS (a National network of reference stations for GPS) in Sweden, SATREF (Satellittbasert Referansesystem) in Norway, and the British Isles GPS archive Facility (BIGF) in the United Kingdom. Figure 1 shows the location of the GPS receivers in Europe. Each GPS receiver provides phase advance and group delay (P-code pseudo-range) data of dual-frequency GPS signals at at least 30-s intervals. TEC obtained from the carrier phase advance is accurate, although it includes an unknown offset because of the unknown initialization constant in phase measurements. In this study, we focus on the perturbation component of TEC, which could be caused by MSTIDs. The perturbation component of the TEC is obtained by subtracting the $1-\mathrm{h}$ running average (average over \pm 30 min centered on the corresponding data) from the original TEC time series for each pair of satellites and receivers. It should be noted that the obtained perturbation 
component of the TEC includes both temporal and spatial variations. The precision of the relative change of TEC is theoretically 0.01-0.02 TECU (where $1 \mathrm{TECU}=10^{16} \mathrm{~m}^{-2}$ ), which corresponds to $1 \%$ of the wavelength of GPS signals L1 (0.19 m) and L2 (0.24 m) (Spilker and Parkinson, 1996).

The TEC is multiplied by a slant factor to convert lineof-sight TEC to a vertical one. The slant factor is defined as $\tau_{0} / \tau_{1}$, where $\tau_{1}$ is the length of the ray path between 250 and $450-\mathrm{km}$ altitudes, and $\tau_{0}$ is the thickness of the ionosphere $(200 \mathrm{~km})$ for the zenith path (Saito et al., 1998). The TEC values are mapped on the ionospheric shell at a $300-\mathrm{km}$ altitude with a pixel size of $0.15^{\circ} \times 0.15^{\circ}$ in latitude and longitude. TEC data with satellite elevation angles of less than $35^{\circ}$ are excluded to reduce the effects of the cycle slips and the uncertainty of location where the TEC is mapped. TEC within a pixel is averaged. To compensate for the scarcity of the TEC data distribution, the TEC value in each pixel is smoothed temporally with a running average of $10 \mathrm{~min}$, during which an ionospheric pierce point (IPP) moves approximately $50 \mathrm{~km}$ around the zenith of a GPS receiver. Then, the TEC map in each epoch is smoothed spatially with the running average within a area covered by 5 pixels in latitude and $5 / \cos \theta$ pixels in longitude, where $\theta$ is latitude. $5 / \cos \theta$ ranges from 5 to 19 between $30^{\circ} \mathrm{N}$ and $75^{\circ} \mathrm{N}$ in latitude. This smoothing technique is the same as that applied to the TEC data in North America by Tsugawa et al. (2007). As a result, TEC maps for perturbation components, covering an area of Europe between $20^{\circ} \mathrm{W}$ and $40^{\circ} \mathrm{E}$ in longitude and between $30^{\circ} \mathrm{N}$ and $75^{\circ} \mathrm{N}$ in latitude, can be obtained with a spatial resolution of approximately $80 \mathrm{~km} \times 80 \mathrm{~km}$ at 30 -s intervals (10-min smoothing). On the TEC perturbation maps, TEC variations with a timescale of 10-60 min can be derived.

We have investigated time sequences of two-dimensional TEC perturbation maps over Europe to detect MSTIDs. Figure $2 \mathrm{a}$ and $\mathrm{b}$ show typical examples of the MSTIDs observed during daytime and nighttime, respectively. Animations of the TEC maps with 30-s resolution during these MSTID events can be viewed in Movies 1 and 2. Wavy structures of the TEC perturbations with phase fronts aligned in the eastwest direction during daytime (Fig. 2a) and in the NW-SE direction during nighttime are recognized in the figures. In this study, we define MSTIDs as the TEC perturbations that satisfy the following criteria in the same way as Kotake et al. (2007) and Otsuka et al. (2011): (1) the TEC perturbation has amplitude exceeding $0.2 \mathrm{TECU}$; (2) the horizontal wavelength of the TEC perturbations is shorter than $1500 \mathrm{~km}$; and (3) the TEC perturbation has more than two phase fronts and propagates on the map. We divide the area of TEC maps almost equally into two regions (higher and lower latitude regions) by $55^{\circ} \mathrm{N}$ in geographic latitude to study latitudinal variations of MSTIDs.

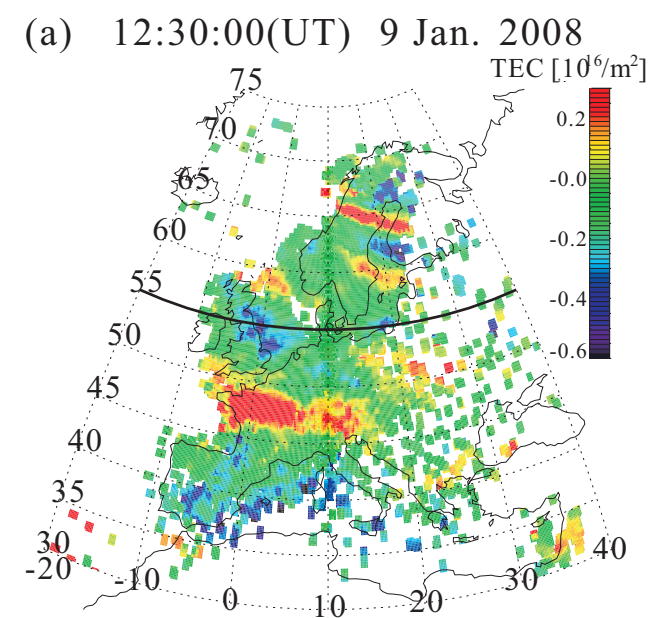

(b) 22:40:00(UT) 17 Aug. 2008

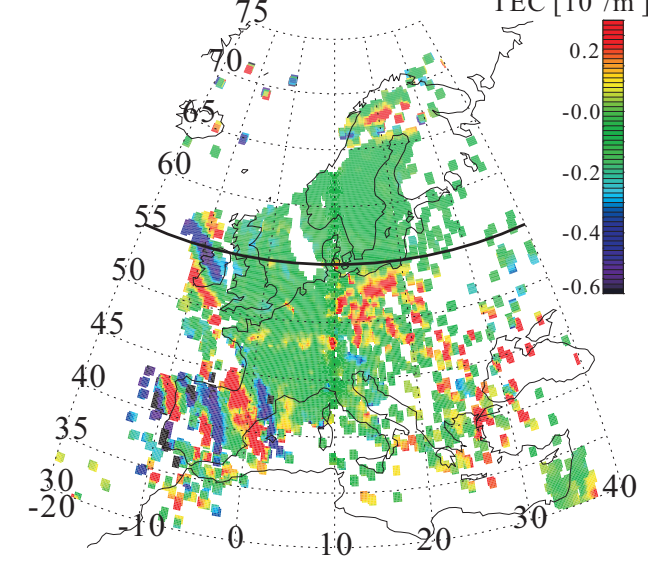

Fig. 2. Two-dimensional maps of TEC perturbations observed with GPS networks in Europe (a) at 12:30 UT on 9 January 2008, and (b) at 22:40 UT on 17 August 2008.

\section{Results}

We have analyzed the TEC data obtained with the GPS receivers in Europe, in 2008, and determined the MSTID occurrence at hourly intervals. We divide all data into hourly and monthly bins and calculate MSTID occurrence rate in each bin. The area of TEC maps is divided almost equally into two regions (higher and lower latitude regions) by $55^{\circ} \mathrm{N}$ in geographic latitude to study latitudinal variations of MSTIDs. Figure 3a and $3 \mathrm{~b}$ show local time and seasonal variations of the MSTID occurrence rate for higher and lower latitudes, respectively. The MSTID occurrence rate is defined as a ratio $M / N$, where $M$ is the number of bins in which MSTID is observed, and $N$ is the number of bins in which GPS TEC data exist. Because the GPS-TEC data can be obtained every day, $N$ is equal to the number of day in each month $(29,30$ or 31$)$. From the figure, we find that the MSTID occurrence rate strongly depends on the season and local time. The occurrence rate is high during daytime 


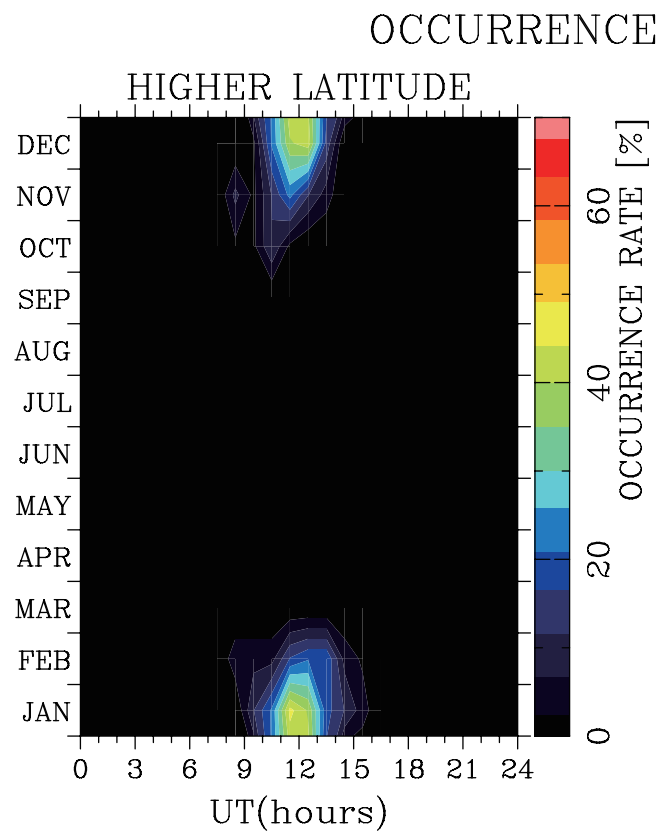

RATE OF MSTID

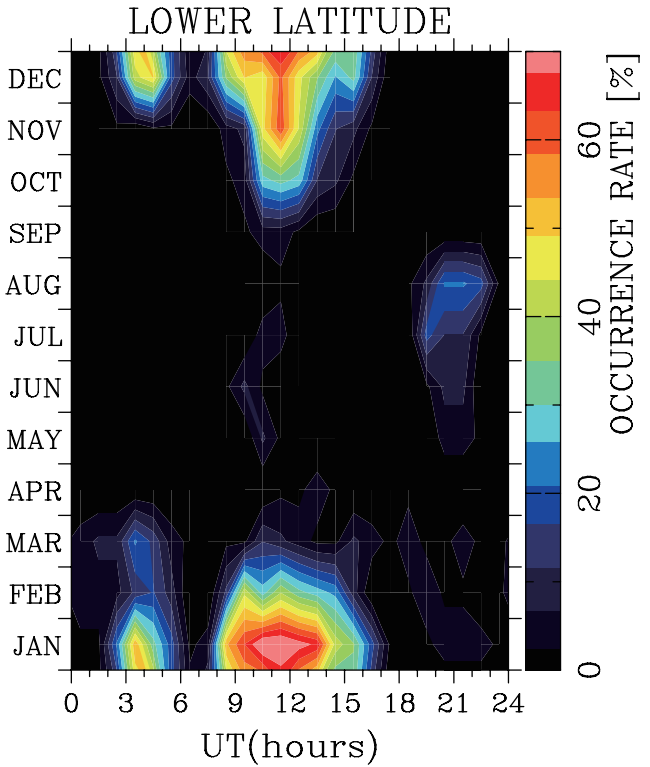

Fig. 3. Local time and seasonal variations in MSTID occurrence rate at (left) higher latitudes $\left(>55^{\circ} \mathrm{N}\right)$ and (right) lower latitudes $\left(<55^{\circ} \mathrm{N}\right)$ of Europe in 2008.
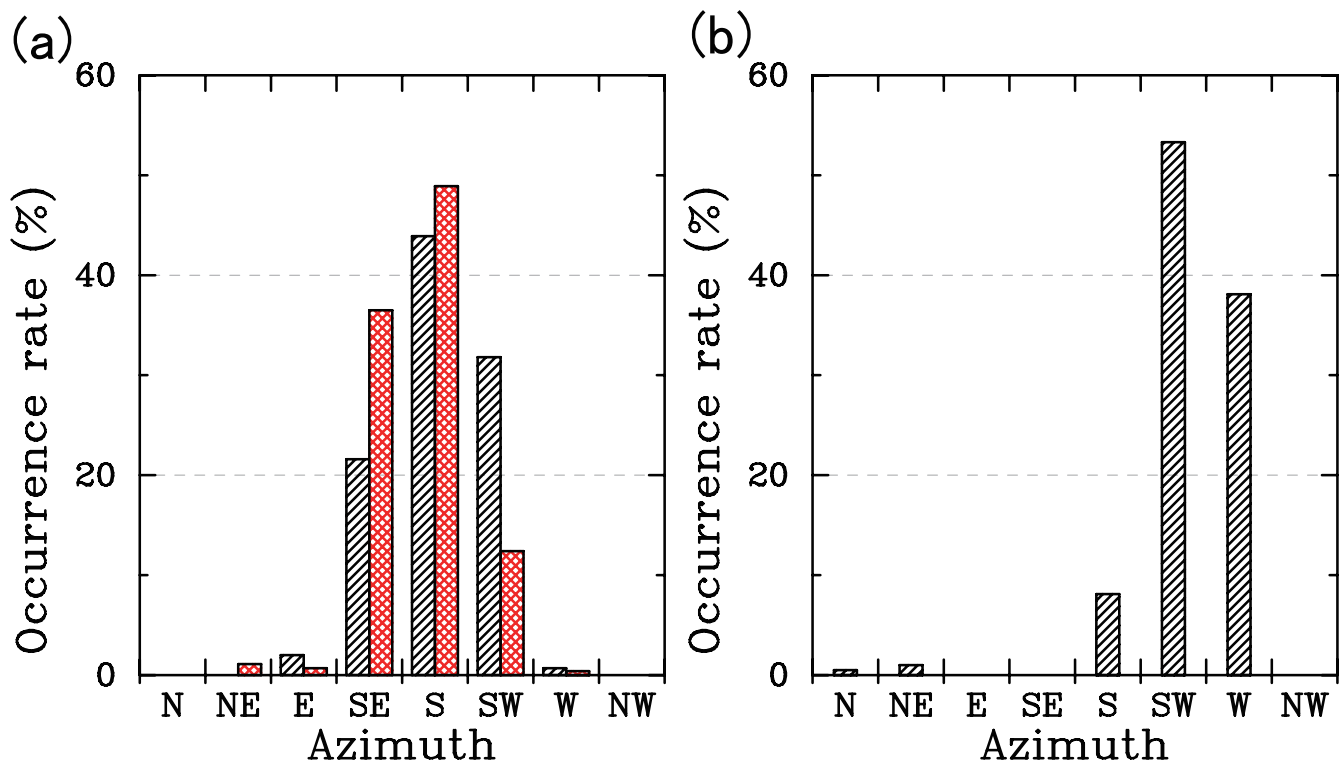

Fig. 4. Propagation direction of the (a) daytime and (b) nighttime MSTIDs at (red) higher and (black) lower latitude regions of Europe in 2008. Note that the nighttime MSTIDs were not observed in the higher latitude region.

(10:00-13:00 UT) in winter at higher latitudes, and during daytime (08:00-16:00) and nighttime (02:00-05:00 UT) in winter at lower latitudes. Also, a secondary peak can be seen around 20:00-22:00 UT in summer at lower latitudes. The latitudinal difference of the nighttime MSTID occurrence is distinct: The maximum of the occurrence rate at lower latitudes is approximately $50 \%$ whereas the occurrence rate at higher latitudes is not discernible. However, the latitudi- nal difference of the daytime MSTID occurrence is not distinct compared to that of the nighttime MSTID occurrence, although it shows a similar tendency: The maximum occurrence rate exceeds $70 \%$ at lower latitudes, whereas it is approximately $45 \%$ at higher latitudes.

The propagation direction of the MSTIDs has also been investigated by using the TEC perturbation maps. Figure 4 shows the propagation direction of the daytime and nighttime 
MSTIDs. We classified all the data into $45^{\circ}$-interval bins and calculated the occurrence rate in each azimuthal bin. During daytime, most of MSTIDs propagate southward. In contrast, the nighttime MSTIDs prefer a southwestward propagation.

\section{Discussion}

By analyzing the GPS TEC data in Europe in 2008, we have disclosed statistical characteristics of occurrence rate and propagation direction of daytime and nighttime MSTIDs. Most of the daytime MSTIDs propagate southward, whereas the nighttime MSTIDs prefer to propagate southwestward. These propagation directions of the daytime and nighttime MSTIDs are consistent with those reported in previous studies (e.g., Jacobson et al., 1995; Kotake et al., 2007; Otsuka et al., 2011; Hernández-Pajares et al., 2006). In these studies, it was suggested that the local time difference of the MSTID propagation direction could arise from corresponding different mechanisms generating MSTIDs.

Daytime MSTIDs over Europe are observed frequently in winter and tend to propagate southward (equatorward). This equatorward propagation is consistent with that reported in previous studies (e.g., Kotake et al., 2006; Evans et al., 1983; Oliver et al., 1997) and is explained below. The MSTIDs have been thought to be caused by neutral gas oscillation caused by atmospheric gravity waves (Hines, 1960; Hooke, 1968). Through the collision of neutral gases with ions in the F-region, the ions move along the geomagnetic field lines $(\boldsymbol{B})$, whereas ion motion across $\boldsymbol{B}$ is restricted because the ion-neutral collision frequency is smaller than the gyrofrequency. This directivity of ion motion causes directivity in the response of the plasma density variations to the gravity waves. The largest-amplitude TEC perturbations could be caused by gravity waves propagating equatorward because the neutral wind oscillation parallel to $\boldsymbol{B}$ is larger for gravity waves propagating equatorward than for those propagating in other directions. Such a directivity in the response of the plasma to the gravity waves could be responsible for the equatorward propagation of the daytime MSTIDs.

In contrast, the nighttime MSTIDs, which propagate southwestward, are considered to be generated by electrodynamical forces including polarization electric fields. Otsuka et al. (2007, 2009) and Makela and Otsuka (2011) have explained the NW-SE phase fronts of the nighttime MSTIDs in the Northern Hemisphere as follows. MSTIDs, which are plasma density perturbations, produce spatial inhomogeneity of the Pedersen conductivity $(\Sigma)$. In the mid-latitude Fregion, the electric current $\boldsymbol{J}$ flows northeastward during nighttime. When a perturbation of $\Sigma$ has a structure elongated from NW to SE, $\boldsymbol{J}$ traverses $\Sigma$. For this case, the polarization electric field $(\boldsymbol{E})$, which is northeastward (southwestward) in the regions of low (high) $\Sigma$, should be generated to maintain a divergence-free $\boldsymbol{J}$. This electric field moves the plasma upward (downward) by $\boldsymbol{E} \times \boldsymbol{B}$ drift, causing the plasma density perturbations. This is a possible mechanism for generating the nighttime MSTIDs with phase fronts elongated from NW to SE in the Northern Hemisphere. Under the condition that the wave vector of $\Sigma$ perturbations lies between the direction of $\boldsymbol{J}$ and the zonal direction, the perturbations in both plasma density and electric field grow with time through the Perkins instability (Perkins, 1973). Consequently, the nighttime MSTIDs observed over Europe could be caused by the electrodynamical forces.

\subsection{Latitudinal difference of MSTIDs}

Our statistical results show that the MSTID occurrence rate is higher at low latitudes than at high latitudes in Europe. In particular, the nighttime MSTID occurrence rate strongly depends on latitude: The nighttime MSTIDs are rarely observed at higher latitudes whereas the occurrence rate reaches a maximum of approximately $50 \%$ at lower latitudes. This latitudinal difference of the MSTIDs may be attributed to the difference between the mechanisms causing the daytime and nighttime MSTIDs. In this subsection, to investigate the effect of the dip angle of the magnetic field on the amplitude of TEC perturbations caused by MSTIDs, we have performed model calculations for the daytime and nighttime MSTIDs at different two dip angles $\left(63.5^{\circ}\right.$ and $\left.74.2^{\circ}\right)$, which are representatives of the lower and higher latitudes of the TEC map studied in this paper. As discussed above, the daytime and nighttime MSTIDs could be generated by atmospheric gravity waves and electric field oscillations, respectively. For the model calculations, the background altitude profiles of the plasma density is taken from the IRI-95 ( International reference ionosphere) model. Similar model calculations have been performed by Shiokawa et al. (2003b).

To simulate the daytime MSTIDs, we have performed model calculations of the plasma density perturbations caused by a gravity wave, which is an oscillation of neutral air. The neutral air moves ions only along the geomagnetic field through neutral-ion collisions. According to Hooke (1968), the plasma density perturbation $N_{\mathrm{e}}^{\prime}$ caused by a gravity wave with angular frequency $\omega$ is given by

$N_{\mathrm{e}}^{\prime}=i \omega^{-1} u_{\|}\left[\frac{\partial N_{\mathrm{e}}}{\partial z} \sin I-i k_{\|} N_{\mathrm{e}}\right]$,

where $i=\sqrt{-1}, u_{\|}$, and $k_{\|}$are the projection of the neutral wind velocity and the wave vector in the direction of the geomagnetic field, respectively, $z$ is the altitude, $I$ is the dip angle of the geomagnetic field, and $N_{\mathrm{e}}$ is the ambient plasma density.

Figure 5a shows the result of the model calculation in the north-south cross section. We consider the gravity wave propagating southward (azimuth of $135^{\circ}$ from the north) with a horizontal wavelength of $300 \mathrm{~km}$ and a period of $40 \mathrm{~min}$. I is set to $63.5^{\circ}$, which corresponds to the lower latitudes studied in this paper. The vertical wavelength is obtained from the gravity-wave dispersion relation. $u_{\|}$is set to be $7.0 \mathrm{~m} \mathrm{~s}^{-1}$ for 

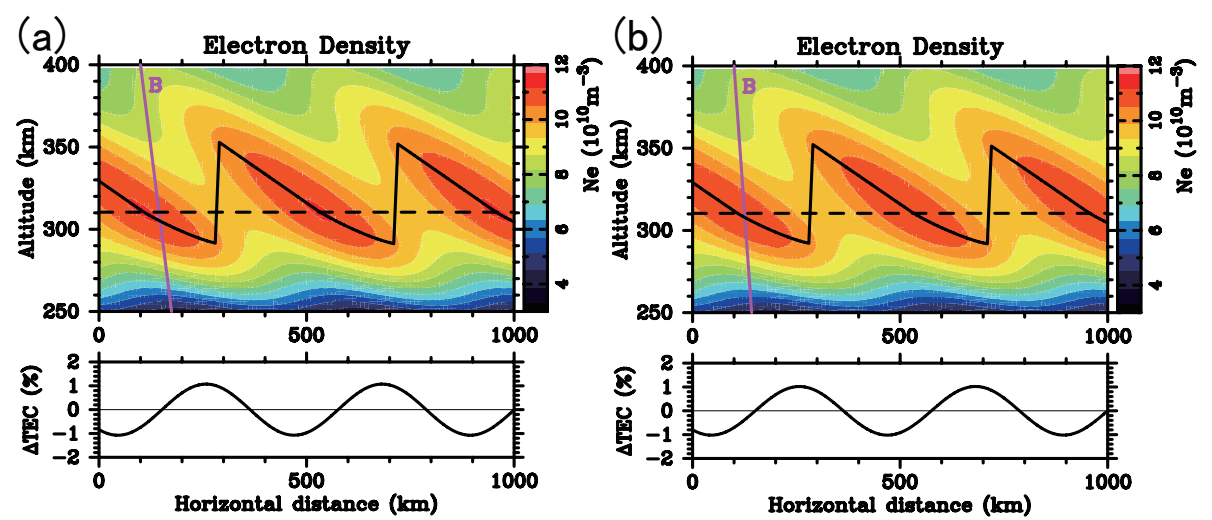

Fig. 5. Model calculations of the plasma density perturbations caused by a gravity wave at dip angles of (a) $63.5^{\circ}$ and (b) $74.2^{\circ}$, respectively. The gravity wave is assumed to propagate southeastward (azimuth of $135^{\circ}$ from the north) with a horizontal wavelength of $300 \mathrm{~km}$ and a period of $40 \mathrm{~min}$, with a neutral wind oscillation amplitude of $7.0 \mathrm{~m} \mathrm{~s}^{-1}$. The color scale, black solid line, and black dashed line in the top panel indicate the plasma density, peak altitude of the electron density, and original peak altitude without the perturbations, respectively. The purple line indicates the dip angle of the magnetic field. The bottom panel shows the amplitude of TEC perturbations as a percentage of the background value. TEC is obtained from integration of the plasma density from 250 to $400 \mathrm{~km}$ in altitude.

Table 1. Model calculation of amplitude in TEC perturbations.

\begin{tabular}{lcc}
\hline Dip angle $(I)$ & $63.5^{\circ}$ & $74.2^{\circ}$ \\
\hline Gravity wave & $1.09 \%$ & $1.04 \%$ \\
Oscillating electric field & $1.01 \%$ & $0.43 \%$ \\
\hline
\end{tabular}

all altitudes. In the figure, the structure of the plasma density perturbations is found to represent that of the gravity wave input in the model. Vertical TEC is obtained by integrating the plasma density from 250 to $400 \mathrm{~km}$ in altitude. We define the vertical TEC with and without the gravity wave as $T_{1}$ and $T_{0}$, respectively. The bottom panel of Fig. 5a shows the perturbation component $\left(T_{1}-T_{0}\right)$ as a percentage to the original TEC $\left(T_{0}\right)$. We find that the amplitude of the TEC perturbation is $1.09 \%$, which is comparable to amplitude of the observed TEC perturbations caused by the MSTIDs. This amplitude is smaller than that of the $N_{\mathrm{e}}$ perturbation, which is larger than $10 \%$, because the $N_{\mathrm{e}}$ perturbations are smeared out by integrating $N_{\mathrm{e}}$.

To investigate the dip angle dependence of the TEC perturbations, we have performed the same model calculation but with a dip angle of $74.2^{\circ}$, which corresponds to the higher latitudes studied in this paper. The result is shown in Fig. 5b. For this case, the amplitude of the TEC perturbation is $1.04 \%$. The results of the model calculations are summarized in Table 1. The amplitude of TEC perturbations does not change significantly with increasing dip angle. This is because phase fronts of the plasma density perturbations, as shown in Fig. 5a and b, are same as that of the gravity wave input in this model calculation.

To simulate the nighttime MSTIDs, we have performed a similar model calculation for the case of oscillating electric fields causing velocity perturbation $\boldsymbol{v}^{\prime}$. Since $\boldsymbol{v}^{\prime}$ is an $\boldsymbol{E} \times \boldsymbol{B}$ drift caused by the polarization electric fields associated with MSTIDs, $\boldsymbol{v}^{\prime}$ is normal to both $\boldsymbol{k}$ and the geomagnetic field. From the continuity equation of ions, in the same way as Shiokawa et al. (2003b), the relationship between the plasma density perturbation $N_{\mathrm{e}}^{\prime}$ and the velocity perturbation $\boldsymbol{v}^{\prime}$ is obtained as follows

$N_{\mathrm{e}}^{\prime}=-i \omega^{-1} \boldsymbol{v}^{\prime} \frac{\partial N_{\mathrm{e}}}{\partial z} \cos I \sin D$

where $D$ is azimuth of the MSTID propagation direction from the magnetic north. The plasma density perturbation $N_{\mathrm{e}}^{\prime}$ is caused by the vertical displacement of $N_{\mathrm{e}}$ so that the amplitude of $N_{\mathrm{e}}^{\prime}$ is proportional to the vertical component of the $\boldsymbol{E} \times \boldsymbol{B}$ drift, that is, $\boldsymbol{v}^{\prime} \cos I \sin D$. We assume that an MSTID with a horizontal wavelength of $300 \mathrm{~km}$ propagates southwestward $\left(D=225^{\circ}\right)$, and simulate $N_{\mathrm{e}}^{\prime}$ caused by an oscillating $\boldsymbol{v}^{\prime}$ that is sinusoidal with an amplitude of $35 \mathrm{~m} \mathrm{~s}^{-1}$. Figure 6 a shows the result of the model calculation for the case of $I=63.5^{\circ}$. Owing to the northward and upward (southward and downward) $\boldsymbol{E} \times \boldsymbol{B}$ drift, a field-aligned structure of the plasma density is formed because the electric field is assumed to be transmitted along the magnetic field without attenuation. The amplitude of the TEC perturbations is $1.01 \%$.

We have performed the same calculation but for the case of $I=74.2^{\circ}$. For this case, the amplitude of the TEC perturbation is $0.58 \%$. We find that the amplitude of the TEC perturbations decreases by almost half by increasing the dip angle from $63.5^{\circ}$ to $74.2^{\circ}$. This is attributed to the latitudinal difference of $\cos I$ in Eq. (2). At increasing latitude, $\cos I$ becomes small, and thus $N_{\mathrm{e}}^{\prime}$ also becomes small. This corresponds to the fact that the $\boldsymbol{E} \times \boldsymbol{B}$ drift velocity becomes nearly horizontal at increasing latitude, so the TEC perturbations are small at high latitudes. 

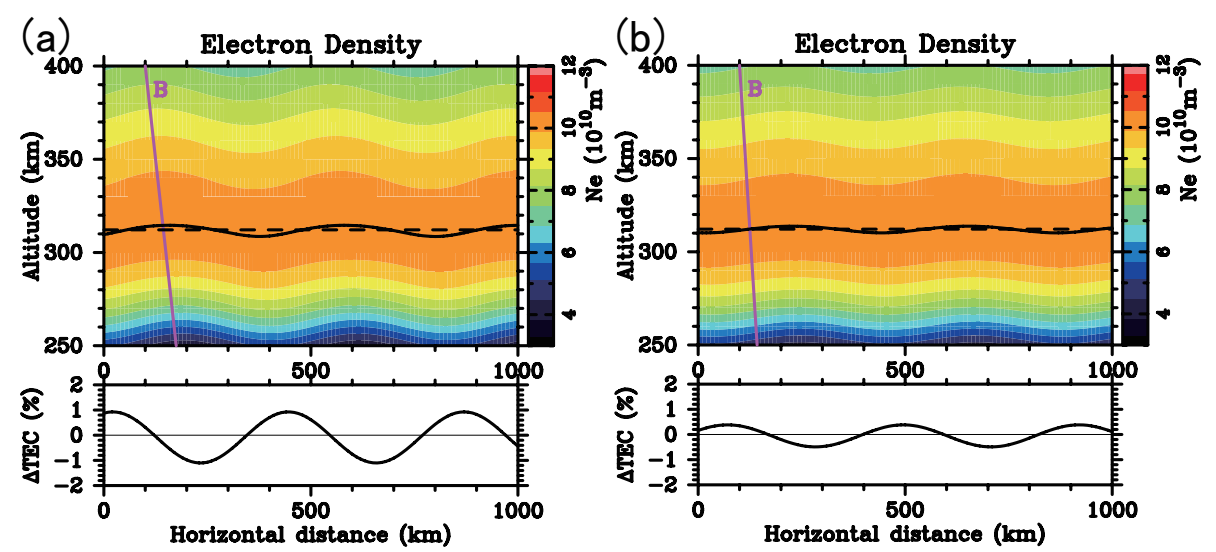

Fig. 6. Same as Fig. 5 but for the plasma density perturbations caused by $\boldsymbol{E} \times \boldsymbol{B}$ drift resulting from the oscillating electric field, which is a sinusoidal perturbation with an amplitude of $35 \mathrm{~m} \mathrm{~s}^{-1}$, propagating southwestward $\left(225^{\circ}\right.$ from the north). The horizontal wavelength and period are $300 \mathrm{~km}$ and $40 \mathrm{~min}$, respectively.

From the model calculations, we find that the amplitude of the TEC perturbations caused by a gravity wave does not show any distinct difference at dip angles of $63.5^{\circ}$ and $74.2^{\circ}$ but that the amplitude of the TEC perturbations caused by an oscillating electric field largely depends on the dip angle. These dip angle differences of the TEC perturbation amplitudes could be responsible for the latitudinal difference of the observed MSTID occurrence rate. The latitudinal difference of the daytime MSTID occurrence rate is small. However, the nighttime MSTID occurrence rate is higher at higher latitudes than at lower latitudes. The difference in the latitudinal dependence of the occurrence rate between the daytime and nighttime MSTIDs could be attributed to the difference of the mechanisms generating the daytime and nighttime MSTIDs.

\subsection{Longitudinal difference of nighttime MSTIDs}

The present paper shows that the occurrence rate of the nighttime MSTIDs over Europe has a primary peak around the December solstice and a secondary peak around the June solstice. Local time of the primary and secondary peaks of the MSTID occurrence is different; the primary peak appears around 02:00-05:00 UT, whereas the secondary peak around 20:00-22:00 UT. By analyzing the GPS TEC data, Hernández-Pajares et al. (2006) have shown that the nighttime MSTIDs over Europe occur most frequently at premidnight around the June solstice. In Fig. 7 of HernándezPajares et al. (2006), high occurrence rate of the nighttime MSTIDs over Europe can be seen after midnight around the December solstice. Both Hernández-Pajares et al. (2006) and the current paper suggest that occurrence rate of the nighttime MSTIDs over Europe shows semiannual variation with maxima in the June and December solstices, although Hernández-Pajares et al. (2006) report that the occurrence rate around the June solstice is larger than the December solstice. On the other hand, Kotake et al. (2007) and Otsuka et al. (2011), who have also analyzed the GPS TEC data in Southern California and Japan, respectively, have shown that the occurrence rate of the nighttime MSTIDs is highest around the June solstice and that it has a secondary peak around the December solstice. These seasonal and longitudinal variations of the nighttime MSTID occurrence rate are consistent with those of the spread-F occurrence rate reported by Bowman (1992). He has suggested that the high occurrence rate of the spread-F at both summer and winter solstices may be a result of the low neutral density at the solstice. The neutral density shows semiannual variation with maxima at equinoxes and minima at solstices. When the neutral density is smaller, the amplitude of gravity waves becomes larger (e.g., Hines, 1960). Gravity waves with largeamplitude neutral wind oscillation could cause large plasma density perturbations, such as MSTIDs and spread-F. Moreover, Kotake et al. (2006) have suggested that the linear growth rate of the Perkins instability, which is a likely source of nighttime MSTIDs, is inversely proportional to the neutral density (Perkins, 1973). These facts suggest that seasonal variation of the nighttime MSTIDs could be attributed to that of the neutral density.

Recent studies suggested that the nighttime MSTIDs could be generated by electrodynamical forces with coupling between E- and F-regions. Tsunoda and Cosgrove (2001) have suggested that intense electric fields generated by the Hall polarization process in the Es layer could be transmitted to the F-region, causing a positive feedback process of the perturbations in the polarization electric fields and plasma density. Cosgrove and Tsunoda (2002) have proposed an Es layer instability mechanism, which operates under the condition that the perturbation of the Es layer plasma density has structures with wavefronts aligned from northwest to southeast. When the Es layer instability is coupled with the Perkins instability that operates in the F-region, the coupling instability 
provides a larger growth rate than either instability alone (Cosgrove and Tsunoda, 2004).

Saito et al. (2007) have reported that day-to-day variation of the nighttime MSTID occurrence is consistent with the field-aligned irregularities (FAIs) embedded in the Es layer. Otsuka et al. (2007) have shown positive correlation of the nighttime MSTID occurrence with the appearance of the Es layer and inhomogeneity of the Es plasma density. They have pointed out that day-to-day variation of the nighttime MSTID occurrence could be controlled by the appearance of the Es layer by assuming that the irregularities and inhomogeneity of the Es layer coincide with the Es layer. According to their idea, seasonal variation of the nighttime MSTID can be explained in terms of the Es layer occurrence, as described below. The Es layer is considered to be formed by the convergence of metallic ions owing to the vertical shear in zonal wind, which is eastward below the Es layer and westward above the Es layer (Whitehead, 1961). Taguchi and Shibata (1961) and Wu et al. (2005) have shown global distribution and seasonal variations of the Es layer plasma density. Their results show that the Es layer occurrence rate is highest in summer and that hemispheric asymmetry and longitudinal dependence exist in the Es plasma density. In the Japanese and Australian longitudinal sector, the Es plasma density is higher over Japan (Northern Hemisphere) around the June solstice than over Australia (Southern Hemisphere) around the December solstice. As described above, in this longitudinal sector, the MSTID occurrence rate shows a semiannual variation with a major peak around the June solstice and a minor peak around the December solstice (Shiokawa et al., 2003a; Kotake et al., 2006; Otsuka et al., 2011). Saito et al. (2007) and Otsuka et al. (2007) have suggested that high plasma density of the Es layer in the Northern Hemisphere, around the June solstice, could be caused by the major peak of MSTID occurrence rate around the June solstice in the Japanese and Australian longitudinal sector, and that the minor peak of the MSTID occurrence rate around the December solstice could be responsible for the Es layer over Australia in the Southern Hemisphere.

The seasonal variations of both the nighttime MSTID and Es layer occurrences depend on longitude. Otsuka et al. (2007) have pointed out that the above scenario regarding the relationship between both the MSTID and Es layer can be applicable to data in the American longitudinal sector, where the Es plasma density is higher in the Southern Hemisphere around the December solstice than in the Northern Hemisphere around the June solstice. Garcia et al. (2000) and Martinis et al. (2010) report that MSTID occurrence rates at Arecibo, Puerto Rico $\left(18.3^{\circ} \mathrm{N}, 66.7^{\circ} \mathrm{W}\right)$, have a major peak around the December solstice. Furthermore, Park et al. (2010), who have analyzed plasma density measured by the CHAMP, KOMPSAT-1, and DMSP satellites have shown that the nighttime MSTID occurrence rate in the Asian/Oceanian (eastern Pacific/American) region is higher during the June (December) solstice. They have sug- gested that the above scenario regarding the relationship between the nighttime MSTID occurrence and the Es layer appearance is applicable globally.

These results suggest that the Es layer plasma density may control longitudinal and seasonal variations of the MSTID occurrence rates. According to this scenario, the MSTID occurrence rate in Europe is predicted to be highest around the June solstice since the Es layer plasma density is higher over Europe (in the Northern Hemisphere) around the June solstice than over Africa (in the Southern Hemisphere) around the December solstice (e.g., Wu et al., 2005). However, our observations show that the occurrence rate of the MSTIDs over Europe is highest around the December solstice. This discrepancy may suggest that the MSTID occurrence is not controlled by the Es layer plasma density alone. As described above, Saito et al. (2007) and Otsuka et al. (2007) have shown positive correlation of the nighttime MSTID occurrence with the appearance of the Es layer and inhomogeneity of the Es plasma density. However, they assume that the inhomogeneity of the Es plasma density coincides with high plasma density in the Es layer. This assumption may not be valid. The inhomogeneity of the Es layer plasma density, which could drive the polarization electric field to keep the current continuity, may play an important role in generating the nighttime MSTIDs. Therefore, the inhomogeneity of the Es layer plasma density may need to be taken into account for understanding the cause of the seasonal/longitudinal variation of the nighttime MSTID occurrence rate.

\section{Summary and conclusions}

Using the densely spaced GPS receiver networks in Europe, we have investigated statistical characteristics of the MSTIDs and have found that the characteristics of the daytime and nighttime MSTIDs are different. The observed characteristics can be summarized as follows:

1. The daytime MSTIDs frequently occur in winter. Its maximum occurrence rate in monthly and hourly bin exceeds $70 \%$ at lower latitudes and it is approximately $45 \%$ at higher latitudes in Europe. Most of the daytime MSTIDs propagate southward. From the preferred propagation direction, we can speculate that the daytime MSTIDs are generated by gravity waves.

2. The nighttime MSTIDs occur frequently in winter with a secondary occurrence peak in summer. Its maximum occurrence rate in monthly and hourly bin is approximately $50 \%$ at lower latitudes, whereas the nighttime MSTIDs were rarely observed at higher latitudes. The nighttime MSTIDs propagate southwestward, on average. The southwestward propagation suggests that electrodynamical forces could play an important role in generating the nighttime MSTIDs. 
3. The occurrence rate of both daytime and nighttime MSTIDs is higher at lower latitudes than at higher latitudes, whereas the occurrence rate of the nighttime MSTIDs shows a more distinct latitudinal difference than that of the daytime MSTIDs.

We have performed model calculations of the plasma density perturbations caused by a gravity wave and an oscillating electric field to reproduce the daytime and nighttime MSTIDs, respectively, over Europe. We find that TEC perturbations caused by gravity waves do not show a dip angle dependence for dip angles between $63.5^{\circ}$ and $74.2^{\circ}$ and that TEC perturbations caused by the oscillating electric field have a larger amplitude at lower latitudes than at higher latitudes. This dip angle dependence in the amplitude of TEC perturbations could be partially responsible for the latitudinal difference of the MSTID occurrence rate.

We have compared the MSTID occurrence rate over Europe with that in other regions and discussed the longitudinal difference of the nighttime MSTID occurrence rate along with the E- and F-region coupling processes. Previous studies suggest that the longitudinal and seasonal variations of the nighttime MSTID occurrence could be attributed to those of the Es layer occurrence rate. However, the seasonal variation of the nighttime MSTID occurrence, as shown in the present paper, is not consistent with this scenario. We speculate that the nighttime MSTID occurrence is not controlled by the Es layer occurrence alone.

\section{Supplementary material related to this article is available online at: http://www.ann-geophys.net/31/163/ 2013/angeo-31-163-2013-supplement.zip.}

Acknowledgements. GPS data were provided by IGS, EPN, BKGE, OLG, IGNE, DUT, ASI, ITACYL, ESEAS, SWEPOS, SATREF, and BIGF.

Topical Editor K. Kauristie thanks S. Saito and one anonymous referee for their help in evaluating this paper.

\section{References}

Bowman, G. G.: Upper atmosphere neutral-particle density variations compared with spread-F occurrence rates at locations around the world, Ann. Geophys., 10, 676-682, 1992.

Cosgrove, R. B. and Tsunoda, R. T.: A direction-dependent instability of sporadic-E layers in the nighttime midlatitude ionosphere, Geophys. Res. Lett., 29, 1864, doi:10.1029/2002GL014669, 2002.

Cosgrove, R. B. and Tsunoda, R. T.: Instability of the $E-F$ coupled nighttime midlatitude ionosphere, J. Geophys. Res., 109, A04305, doi:10.1029/2003JA010243, 2004.

Evans, J. V., Holt, J. M., and Wand, R. H.: A differential-Doppler study of traveling ionospheric disturbances from Millstone Hill, Radio Sci., 18, 435-451, 1983.
Garcia, F. J., Kelley, M. C., Makela, J. J., and Huang, C. S.: Airglow observations of mesoscale low-velocity traveling traveling ionospheric disturbances at midlatitudes, J. Geophys. Res., 105, 18407-18415, 2000.

Hernaández-Pajares, M., Juan, J. M., and Sanz, J.: Medium-scale traveling ionospheric disturbances affecting GPS measurements: Spatial and temporal analysis, J. Geophys. Res., 111, A07S11, doi:10.1029/2005JA011474, 2006.

Hines, C. O.: Internal atmospheric gravity waves at ionospheric heights, Can. J. Phys., 38, 1441-1481, 1960.

Hocke, K. and Schlegel, K.: A review of atmospheric gravity waves and travelling ionospheric disturbances: 1982-1995, Ann. Geophys., 14, 917-940, doi:10.1007/s00585-996-0917-6, 1996.

Hooke, W. H.: Ionospheric irregularities produced by internal atmospheric gravity waves, J. Atoms. Terr. Phys., 38, 1441-1481, 1968.

Hunsucker, R.: Atmospheric gravity waves generated in the highlatitude ionosphere: A review, Rev. Geophys. Space Phys., 20, 293-315, 1982.

Jacobson, A. R., Carlos, R. C., Massey, R. S., and Wu, G.: Observations of traveling ionospheric disturbances with a satellitebeacon radio interferometer: Seasonal and local time behavior, J. Geophys. Res., 100, 1653-1665, 1995.

Kelley M. C. and Makela, J. J.: Resolution of the discrepancy between experiment and theory of midlatitude F-region structures Geophys. Res. Lett., 28, 2589-2592, 2001.

Kelley, M. C. and Miller, C. A.: Electrodynamics of midlatitude spread F, 3. Electrohydrodynamic waves? A new look at the role of electric fields in thermospheric wave dynamics, J. Geophys. Res., 102, 11539-11547, 1997.

Kelley, M. C., Haldoupis, C., Nicolls, M. J., Makela, J. J., Belehaki, A., Shalimov, S., and Wong, V. K.: Case studies of coupling between the $\mathrm{E}$ and $\mathrm{F}$ regions during unstable sporadic-E conditions, J. Geophys. Res., 108, 1447, doi:10.1029/2003JA009955, 2003.

Kotake, N., Otsuka, Y., Tsugawa, T., Ogawa, T., and Saito, A.: Climatological study of GPS total electron content variations caused by medium-scale traveling ionospheric disturbances, J. Geophys. Res., 111, A04306, doi:10.1029/2005JA011418, 2006.

Kotake, N., Otsuka, Y., Tsugawa, T., Ogawa, T., and Saito, A.: Statistical study of medium-scale traveling ionospheric disturbances observed with the GPS networks in Southern California, Earth Planets Space, 59, 95-102, 2007.

Makela, J. and Otsuka, Y.: Overview of nighttime ionospheric instabilities at low- and mid-latitudes: coupling aspects resulting in structuring at the mesoscale, Space Sci. Rev., 168, 419-440, doi:10.1007/s11214-011-9816-6, 2011.

Martinis, C., Baumgardner, J., Wroten, J., and Mendillo, M.: Seasonal dependence of MSTIDs obtained from $630.0 \mathrm{~nm}$ airglow imaging at Arecibo, Geophys. Res. Lett., 37, L11103, doi:10.1029/2010GL043569, 2010.

Miller, C. A., Swartz, W. E., Kelley, M. C., Mendillo, M., Nottingham, D., Scali, J., and Reinisch, B.: Electrodynamics of midlatitude spread F, 1. Observations of unstable, gravity wave-induced ionospheric electric fields at tropical latitudes, J. Geophys. Res., 102, 11521-11532, 1997.

Ogawa, T., Balan, N., Otsuka, Y., Shiokawa, K., Ihara, C., Shimomai, T., and Saito, A.: Observations and modeling of $630 \mathrm{~nm}$ airglow and total electron content associated with traveling ionospheric disturbances over Shigaraki, Japan, Earth Planets Space, 
54, 45-56, 2002.

Oliver, W. L., Otsuka, Y., Sato, M., Takami, T., and Fukao, S.: A climatology of F-region gravity wave propagation over the middle and upper atmosphere radar, J. Geophys. Res. 102, 1449914512, 1997.

Otsuka, Y., Onoma, F., Shiokawa, K., Ogawa, T., Yamamoto, M., and Fukao, S.: Simultaneous observations of nighttime medium-scale traveling ionospheric disturbances and E-region field-aligned irregularities at midlatitude, J. Geophys. Res., 112, A06317, doi:10.1029/2005JA011548, 2007.

Otsuka, Y., Shiokawa, K., Ogawa, T., Yokoyama, T., and Yamamoto, M.: Spatial relationship of nighttime medium-scale traveling ionospheric disturbances and $\mathrm{F}$ region field-aligned irregularities observed with two spaced all-sky airglow imagers and the middle and upper atmosphere radar, J. Geophys. Res., 114, A05302, doi:10.1029/2008JA013902, 2009.

Otsuka, Y., Kotake, N., Shiokawa, K., Ogawa, T., Tsugawa, T., and Saito, A.: Statistical Study of Medium-Scale Traveling Ionospheric Disturbances Observed with a GPS Receiver Network in Japan, Aeronomy of the Earth's Atmosphere and Ionosphere, IAGA Special Sopron Book Series, Vol. 2, Part 3, 291-299, doi:10.1007/978-94-007-0326-1_21, 2011.

Park, J., Lühr, H., Min, K. W., and Lee, J. J.: Plasma density undulations in the nighttime mid-latitude F-region as observed by CHAMP, KOMPSAT-1, and DMSP F15, J. Atmos. SolarTerr. Phys., 60, 183-192, 2010.

Perkins, F.: Spread F and ionospheric currents, J. Geophys. Res., 78, 218-226, 1973.

Saito, A., Miyazaki, S., and Fukao, S.: High resolution mapping of TEC perturbations with the GSI GPS network over Japan, Geophys. Res. Lett., 25, 3079-3082, 1998.

Saito, S., Yamamoto, M., Hashiguchi, H., Maegawa, A., and Saito, A.: Observational evidence of coupling between quasi-periodic echoes and medium scale traveling ionospheric disturbances, Ann. Geophys., 25, 2185-2194, doi:10.5194/angeo-25-21852007, 2007.
Shiokawa, K., Ihara, C., Otsuka, Y., and Ogawa, T.: Statistical study of nighttime medium-scale traveling ionospheric disturbances using midlatitude airglow imagers, J. Geophys. Res., 108, 1052, doi:10.1029/2002JA009491, 2003a.

Shiokawa, K., Otsuka, Y., Ihara, C., Ogawa, T., and Rich, F. J.: Ground and satellite observations of nighttime medium-scale traveling ionospheric disturbance at midlatitude, J. Geophys. Res., 108, 1145, doi:10.1029/2002JA009639, 2003b.

Spilker, J. J. and Parkinson, B. W.: Overview of GPS operation and design, in: Global Positioning System: Theory and Applications, Vol. 1, pp. 29-56, Am. Inst. of Aeronaut. and Astronaut., Reston, Va, 1996.

Taguchi, S. and Shibata, H.: World maps of $f_{o} E_{S}$. J. Radio Res. Lab., 8, 355-386, 1961.

Tsugawa, T., Otsuka, Y., Coster, A. J., and Saito, A.: Medium-scale traveling ionospheric disturbances detected with dense and wide TEC maps over North America, Geophys. Res. Lett., 34, L22101, doi:10.1029/2007GL031663, 2007.

Tsunoda, R. T. and Cosgrove, R. B.: Coupled electrodynamics in the nighttime midlatitude ionosphere, Geophys. Res. Lett., 28, 4171-4174, 2001.

Whitehead, J. D.: The formation of the sporadic-E layer in the temperate zones, J. Atmos. Terr. Phys., 20, 49-58, 1961.

Wu, D. L., Ao, C. O., Hajj, G. A., de la Torre Juarez, M., and Mannucci, A. J.: Sporadic $E$ morphology from GPSCHAMP radio occultation. J. Geophys. Res., 110, A01306, doi:10.1029/2004JA010701, 2005.

Yokoyama, T. and Hysell, D. L.: A new midlatitude ionosphere electrodynamics coupling model (MIECO): latitudinal dependence and propagation of mediumscale traveling ionospheric disturbances, Geophys. Res. Lett. 37, L08105, doi:10.1029/2010GL042598, 2010.

Yokoyama, T., Hysell, D., Otsuka, Y., and Yamamoto, M.: Threedimensional simulation of the coupled Perkins and Es-layer instabilities in the nighttime midlatitude ionosphere, J. Geophys. Res., 114, A03308, doi:10.1029/2008JA013789, 2009. 